\title{
THE STATIONARY G/G/s QUEUE WITH NON-IDENTICAL SERVERS
}

\author{
PIERRE LE GALL \\ France Telecom, CNET \\ 4 Parc de la Bérengère \\ F-92210 Saint-Cloud, France
}

(Received February, 1998; Revised April, 1998)

\begin{abstract}
We extend a recently developed factorization method to the case of the G/G/s queue with non-identical servers, by presenting three simple properties which lead to a simple numerical calculation method. We compare our results with those determined by classical Markovian (phase) methods in the case of the symmetrical M/G/s queue, and for the mean queueing delay we compare with results given by traffic simulation.
\end{abstract}

Key words: G/G/s Queue, GI/G/s Queue, First Come-First Served, Factorization, Singular Points.

AMS subject classifications: $60 \mathrm{~K} 25,90 \mathrm{~B} 22$.

\section{Introduction}

In a recent paper [3], we studied the stationary $\mathrm{G} / \mathrm{G} / \mathrm{s}$ queue by means of a new factorization method more general than a Wiener-Hopf type of decomposition. In Section 3 , we show how this method may be readily extended to the case of non-identical servers for delayed customers. In Section 4, we then show how the effect of the busy period can be combined with that of the partial occupancies to evaluate the probability of delay. The calculations are made in both Sections 3 and 4 with the aim of deriving a simple numerical calculation method offered by three simple properties. We apply the results successively to the stationary GI/G/s queue and the M/G/s queue. In Section 5, we close with numerical comparisons of results obtained by applying classical Markovian methods to the symmetrical $\mathrm{M} / \mathrm{E}_{2} / \mathrm{s}$ and $\mathrm{M} / \mathrm{H}_{2} / \mathrm{s}$ queues, and we compare the calculated values of the mean queueing delay with results given by traffic simulation.

We begin, in Section 2, by defining notation and assumptions and by outlining the recently-derived preliminary results for the case of the symmetrical $G / G / s$ queue. 


\section{Notation, Assumptions and Preliminary Results for the Symmetric G/G/s Queue}

\subsection{Notation and Assumptions}

Except for the service time distribution, different for each server, the notations and assumptions will be the same as in Le Gall [3]. We consider a queue handled by a multiserver of $\mathbf{s}$ non-identical servers.

a) The Arrival Process: We assume a metrically transitive, strictly stationary process of successive non-negative interarrival times. Let $N(t)$ denote the random number of arrivals in the interval $(0, t]$. We write $d N(t)=1$ or 0 depending on whether or not there is an arrival in the elementary interval $(t, t+d t)$. We exclude the possibility of simultaneous arrivals. We can then write

$$
\mathrm{E}\left\{d N\left(t_{0}\right) \cdot d N\left(t_{0}+t\right)\right\}=\mathrm{E}\left[d N\left(t_{0}\right)\right] \cdot \rho(t) \cdot d t
$$

where $\rho(t)$ is the arrival rate at time $t+t_{0}$ if an arbitrary arrival occurred at time $t_{0}$. We let

$$
\int_{0}^{\infty} e^{z t} \cdot \rho(t) \cdot d t=\alpha_{1}(z)=\sum_{x=1}^{\infty} \varphi_{0, x}(z), \quad \operatorname{Re}(z)<0
$$

where $\varphi_{0, x}(z)$ corresponds to the $x$ th arrival following the epoch $t_{0}$. However, the stationary assumption and the Abelian theorem give that $\lim _{z \rightarrow 0} z \cdot \alpha_{1}(z)=\boldsymbol{\Lambda}$, where $\boldsymbol{\Lambda}$ is the mean arrival rate. In a more general way, we may write for $j=1,2, \ldots$

$$
\begin{aligned}
\mathrm{E}\left\{d N\left(t_{0}\right) \cdot\right. & \left.d N\left(t_{0}+t_{1}\right) \ldots d N\left(t_{0}+t_{1}+\ldots+t_{j-1}+t_{j}\right)\right\} \\
& =\left[d N\left(t_{0}\right)\right] \cdot \boldsymbol{f}_{\boldsymbol{j}}\left(\boldsymbol{t}_{\mathbf{1}} \ldots \boldsymbol{t}_{\boldsymbol{j}}\right) \cdot d t_{1} \ldots d t_{j}
\end{aligned}
$$

and for $\operatorname{Re}\left(z_{j}\right)<0$ and $j=1,2, \ldots$

$$
\int_{0}^{\infty} e^{z_{1} t_{1}} \cdot d t_{1} \ldots \int_{0}^{\infty} e^{z} t^{t} j \cdot d t_{j} \cdot f_{j}\left(t_{1} \ldots t_{j}\right)=\alpha_{j}\left(z_{1} \ldots z_{j}\right)
$$

In the case of a renewal process, the successive arrival intervals $\boldsymbol{Y}_{\boldsymbol{n}}$ are mutually independent and identically distributed, and we let $\varphi_{0}(z)=\mathrm{E}\left[e^{z Y} n\right]$, for $\operatorname{Re}(z)<0$.

Expression (2) becomes

$$
\alpha_{1}(z)=\frac{\varphi_{0}(z)}{1-\varphi_{0}(z)}
$$

and expression (4) becomes

$$
\alpha_{j}\left(z_{j} \ldots z_{j}\right)=\alpha_{1}\left(z_{1}\right) \ldots \alpha_{1}\left(z_{j}\right)
$$

In fact, we assume $\varphi_{0}(z)$ to be holomorphic at the origin. From Paul Levy's theorem, we deduce that $\varphi_{0}(z)$ exists for $\operatorname{Re}(z)<\delta$ where $\delta$ is a positive real number. 
b) The Service Times: The successive service times $\boldsymbol{T}_{\boldsymbol{n}}$ are mutually independent and independent of the arrival process. For server $j(j=1, \ldots, s)$ the service times $\boldsymbol{T}_{\boldsymbol{n}}(j)$ are identically distributed with a distribution function $\boldsymbol{F}_{\mathbf{1}}(\boldsymbol{t} ; \boldsymbol{j})$; and we let $\varphi_{1}(z ; j)=\mathrm{E}\left[e^{z T} n^{(j)}\right]$, for $\operatorname{Re}(z)<0$. We exclude the possibility of batch service and, consequently,

$$
F_{1}(0 ; j)=F_{1}(+0 ; j)=0 .
$$

We assume $\varphi_{1}(z ; j)$ to be holomorphic at the origin. From Paul Levy's theorem, we deduce that $\varphi_{1}(z ; j)$ exists for $\operatorname{Re}(z) \leq \delta$, where $\delta$ is a real positive number.

c) The Service Discipline: The servers are supposed to be non-identical with different service time distributions. But they are indistinguishable for the service discipline which is "first come-first served."

d) The Traffic Handled: Loynes [4] demonstrated the existence of the stationary regime. In Section 3, we shall see that the non-identical servers are equivalent (during the busy period in the stationary regime) to different single servers handling the same value $\boldsymbol{\eta}$ for the traffic intensity per server (at any period), with the necessary and sufficient condition:

$$
\eta<1
$$

e) Queueing Delay: Since the term "waiting time" means "sojourn time" in Little's formula, for clarity we prefer to use the term "queueing delay" $\tau$ for the queueing process only and for an arbitrary customer.

f) Contour Integrals: In this paper we use (Cauchy) contour integrals along the imaginary axis in the complex plane. If the contour (followed from the bottom to the top) is to the right of the imaginary axis (the contour being closed at infinity to the right), we write $\int_{+0}$. If the contour is to the left of the imaginary axis, we write $\int_{-0}$. Unless it is necessary to specify whether the contour is to the right or to the left of the imaginary axis, we write $\int_{0}$.

\subsection{Preliminary Results for the Symmetrical G/G/s Queue}

Now, we outline the recent results that were presented in [3] for the case of the symmetrical $\mathrm{G} / \mathrm{G} / \mathrm{s}$ queue in equilibrium. To avoid very complicated calculations, Le Gall defined the singular points of the function $\mathbf{E}\left[\boldsymbol{e}^{-\boldsymbol{q} \tau}\right]$, with $\operatorname{Re}(q)>0$, relating to the queueing delay $\tau$ for an arbitrary delayed customer. Secondly, Le Gall established conditions under which this function is holomorphic, these conditions being satisfied by a more general factorization method than the Wiener-Hopf type of decomposition. The results will very easily enable one to tackle the difficult case of non-identical servers for the evaluation of the queueing delays of delayed customers.

\subsubsection{The singular points}

For a delayed customer, the queueing delay in server $j$ is denoted $w(j)$. The queueing delay $\tau$ of this customer is 


$$
\tau=\operatorname{Min}^{+}[w(1), \ldots, w(s)]=\operatorname{Max}[0, \min (w(1), \ldots, w(s))]
$$

From an expression given by Pollaczek [6], we may write for $\operatorname{Re}(q) \geq 0$

$$
\left\{\begin{array}{l}
e^{-q \tau}=1-\frac{1}{(2 \pi i)^{s}} \cdot \int_{+0} \exp \left(z_{1} \cdot w(1)\right) \cdot \frac{d z_{1}}{z_{1}} \ldots \int_{+0} \exp \left(z_{s} \cdot w(s)\right) \cdot \frac{d z_{s}}{z_{s}} \cdot \frac{q}{q+\sum_{\nu=1}^{s} z_{\nu}} \\
\text { with } \operatorname{Re}\left(q+\sum_{\nu=1}^{s} z_{\nu}\right)>0
\end{array}\right.
$$

In Le Gall [3], for the symmetrical case, Theorem 1 gives the singular points of the function $\mathrm{E}\left[e^{-q \tau}\right]$. These are the singular points of the following function with $\operatorname{Re}(q)<0$, not holomorphic for $\operatorname{Re}(q)>0$ :

$$
\left\{\begin{array}{l}
\boldsymbol{G}_{\boldsymbol{s}}(q)=1-\frac{1}{(2 \pi i)^{s}} \cdot \int_{+0} \frac{d z_{1}}{z_{1}} \ldots \int_{+0} \frac{d z_{s}}{z_{s}} \cdot \frac{q}{q+\sum_{\nu=1}^{s} z_{\nu}} \cdot \frac{1}{\boldsymbol{R}_{s}\left(z_{1} \ldots z_{s}, q\right)} \\
\text { with } \operatorname{Re}\left(q+\sum_{\nu=1}^{s} z_{\nu}\right)>0
\end{array}\right.
$$

and

$$
\boldsymbol{R}_{\boldsymbol{s}}\left(z_{1} \ldots z_{\boldsymbol{s}} ; \boldsymbol{q}\right)=1+\sum_{\lambda=0}^{s-1}\left(\begin{array}{l}
s \\
\lambda
\end{array}\right) \cdot(-1)^{s-\lambda} \cdot \alpha_{s-\lambda}(q \ldots q) \cdot \prod_{j=\lambda+1}^{s}\left[\varphi_{1}\left(z_{j}\right)-1\right]
$$

where $\alpha_{s-\lambda}(q \ldots q)$ is defined by expression (4). The physical meaning may be perceived in the GI/G/s case, where, due to expression (6), we may write

$$
\boldsymbol{R}_{s}\left(z_{1} \ldots z_{s} ; q\right)=1+\sum_{\lambda=0}^{s-1}\left(\begin{array}{l}
s \\
\lambda
\end{array}\right) \cdot(-1)^{s-\lambda} \cdot\left[\alpha_{1}(q)\right]^{s-\lambda} \cdot \prod_{j=\lambda+1}^{s}\left[\varphi_{1}\left(z_{j}\right)-1\right]
$$

or more simply

$$
R_{s}\left(z_{1} \ldots z_{s} ; q\right)=\prod_{j=1}^{s}\left[1-\alpha_{1}(q) \cdot\left(\varphi_{1}\left(z_{j}\right)-1\right)\right]
$$

The case $\boldsymbol{j}$ corresponds to the server $\boldsymbol{j}$, assumed to be in isolation with an arrival process corresponding to $\alpha_{1}(q)$. For the $\mathrm{G} / \mathrm{G} / \mathrm{s}$ queue, instead of $\boldsymbol{R}_{\boldsymbol{s}}$, we want to define a holomorphic function $V_{s}\left(z_{1} \ldots z_{s} ; q\right)$ for $\operatorname{Re}\left(z_{j}\right) \geq 0(j=1, \ldots, s)$ and $\operatorname{Re}(q) \geq 0$, so that $\boldsymbol{V}_{\boldsymbol{s}}(\mathbf{0} \ldots \mathbf{0} ; \boldsymbol{q})$ has the same singular points as the function $\boldsymbol{G}_{\boldsymbol{s}}(\boldsymbol{q})$ given by expressions (10) and (11).

\subsubsection{The factorization method}

Let

$$
V_{s}\left(z_{1} \ldots z_{s} ; q\right)=1-U_{s}\left(z_{1} \ldots z_{s} ; q\right)
$$


In Le Gall [3] for the symmetrical case, we proved that the function $U_{s}$ has to satisfy the following conditions of factorization:

We set

$$
\boldsymbol{U}_{\boldsymbol{s}}\left(z_{1} \ldots z_{\boldsymbol{s}} ; \boldsymbol{q}\right)=\frac{(-1)^{s} \cdot \alpha_{\boldsymbol{s}}(q \ldots q) \cdot \prod_{j=1}^{s}\left[\varphi_{1}\left(z_{j}\right)-1\right]}{\boldsymbol{R}_{\boldsymbol{s}}\left(z_{1} \ldots z_{\boldsymbol{s}}, \boldsymbol{q}\right)} \cdot \prod_{i=1}^{s} M_{i}\left(z_{1} \ldots z_{s} ; q\right)
$$

where $\boldsymbol{R}_{\boldsymbol{s}}$ is defined by expression (11) and $\boldsymbol{U}_{\boldsymbol{s}}$ is holomorphic for $\operatorname{Re}\left(z_{i}\right) \geq 0$ $(i=1, \ldots, s)$ and $\operatorname{Re}(q) \geq 0$, with the following conditions for $\boldsymbol{M}_{\boldsymbol{i}}$ :

a) $\quad \boldsymbol{M}_{\boldsymbol{i}}$ is holomorphic for $\operatorname{Re}\left(z_{i}\right)<0, i=1, \ldots, s$;

b) $\quad M_{i}\left(z_{1} \ldots z_{i-1},-q-\sum_{\nu=1}^{i-1} z_{\nu}, z_{i+1} \ldots z_{s} ; q\right) \equiv 1$.

Then, we have

$$
V_{s}(0 \ldots 0 ; q)=G_{s}(q)
$$

where $\boldsymbol{G}_{\boldsymbol{s}}(\boldsymbol{q})$ is given by expressions (10) and (11).

Note the following facts.

1) Factorization (14) is still valid when we substitute

$$
\varphi_{1}\left(z_{j} ; j\right) \text { for } \varphi_{1}\left(z_{j}\right)
$$

in expressions (11) and (14), where $\varphi_{1}\left(z_{j} ; j\right)$ has been defined in subsection (2.1.b).

2) To establish this factorization method, we had to use expression (22) in Le Gall [3] for $R(q) \geq 0$ :

$$
U_{s}(0 \ldots .0 ; q)=\frac{(-1)^{s}}{(2 \pi i)^{s}} \cdot \int_{-0} \frac{d z_{1}}{z_{1}} \ldots \int_{-0} \frac{d z_{s}}{z_{s}} \cdot \frac{q}{q+\sum_{\nu=1}^{s} z_{\nu}} \cdot U_{s}\left(z_{1} \ldots z_{s} ; q\right) .
$$

\subsubsection{The function $U_{s}$}

For the symmetrical $\mathrm{G} / \mathrm{G} / \mathrm{s}$ queue, the function $\boldsymbol{U}_{\boldsymbol{s}}$ has been defined in Le Gall [3]:

$$
\begin{aligned}
& U_{s}\left(z_{1} \ldots z_{s} ; q\right)=\operatorname{Exp}\left\{\frac{-1}{(2 \pi i)^{s}} \cdot \int_{-0}\left[\frac{1}{q+\zeta_{1}}+\frac{1}{z_{1}-\zeta_{1}}\right] d \zeta_{1}\right. \\
& \left.\ldots \int_{-0}\left[\frac{1}{q+\sum_{\nu=1}^{s-1} z_{\nu}+\zeta_{s}}+\frac{1}{z_{s}-\zeta_{s}}\right] d \zeta_{s} \cdot \log N_{s}\left\{\zeta_{1} \ldots \zeta_{s}\right)\right\}
\end{aligned}
$$

with

$$
R\left(q+\sum_{\nu=1}^{i-1} z_{\nu}+\zeta_{i}\right)>0, \quad i=1, \ldots, s .
$$

The function $N_{s}$ is given by 


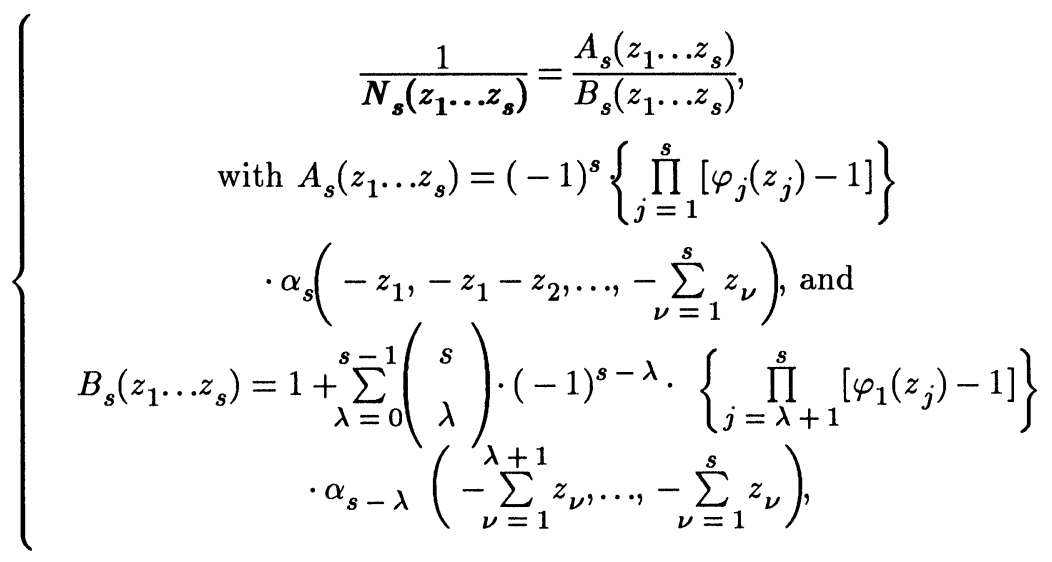

where $\alpha_{j}\left(z_{1} \ldots z_{j}\right)$ is defined by expression (4).

\section{The Delayed Customer}

In this section, we consider the busy period only and a server's behavior during this period (= congestion state). There is generated (for server $j$ ) a queueing delay $\boldsymbol{w}(\boldsymbol{j})$ for $j=1, \ldots, s$, while the multiserver generates a queueing delay $\tau$ for an arbitrary delayed customer, in a stationary regime.

\subsection{The Traffic Per Server}

Let $\boldsymbol{T}(\boldsymbol{j})$ denote the service time of an arbitrary customer in $\operatorname{server} \boldsymbol{j}(\boldsymbol{j}=1, \ldots, s)$, in a stationary regime. The termination rate is $\mu_{j}=(1 / T(j))$. The total termination rate for the multiserver (during a busy period in the steady state) is

$$
\boldsymbol{\mu}=\sum_{j=1}^{s} \mu_{j}=\left(\frac{s}{\bar{T}}\right)
$$

defining the mean service time $\overline{\boldsymbol{T}}$ of the multiserver. Let

$$
\mu_{j}=\frac{\mu}{k_{j}} \quad \text { with } \sum_{j=1}^{s} \frac{1}{k_{j}}=1 .
$$

In other words, $\boldsymbol{k}_{\boldsymbol{j}}(>0)$ is defined by the relation

$$
\overline{T(j)}=k_{j} \cdot\left(\frac{\bar{T}}{s}\right)
$$

The total arrival rate is $\lambda=[\mathrm{E} d N(t)]$. The traffic intensity handled by the multiserver is $\lambda \cdot \bar{T}=[\mathrm{E} d N(t)] \cdot \bar{T}$. Due to the stationary regime and expression (20) for $\boldsymbol{\mu}_{\boldsymbol{j}}$, the arrival rate at server $\boldsymbol{j}$ is

$$
\left.\lambda_{j}=\frac{\lambda}{k_{j}}=\frac{1}{k_{j}} \cdot \mathrm{E} d N(t)\right]
$$

The traffic intensity handled by server $\boldsymbol{j}$ is, due to expressions (21) and (22),

$$
\eta_{j}=\lambda_{j} \cdot \overline{T(j)}=\frac{1}{s} \cdot(\lambda \cdot \bar{T})=\eta
$$


This traffic intensity is the same in each server. We may conclude that the following property holds.

Property 1 (Behavior of server $j$ for the non-symmetrical G/G/s queue in a stationary regime): Server $j$ behaves as a $G / G / 1$ server, as if an arbitrary arrival is chosen with probability $\left(1 / k_{j}\right)$ of being handled by server $\boldsymbol{j}$. It follows that the traffic intensity $\boldsymbol{\eta}$ has the same value in each server during a busy period, in the steady state.

Note the following facts.

1) The symmetrical $G / D / s$ queue has to be excluded because of a deterministic mechanism for the choice of arrivals. But, when the service times are nonidentical, the above property is correct.

2) For the symmetrical $G / G / s$ queue, we have

$$
\boldsymbol{k}_{j}=s, j=1, \ldots, s
$$

We deduce the queueing delay $\tau$ of an arbitrary delayed customer in the G/G/s queue by applying the expectation operator to expression (9) to evaluate $\mathrm{E}\left[e^{-q \tau}\right]$. But, we want to check in the case when $\boldsymbol{k}_{\boldsymbol{j}}$ is valued in set $(1,2, \ldots, s)$.

\subsection{The Distribution of the Queueing Delay}

Expressions (9) and (10) may be applied immediately. For an arrival process, being renewal, expression (12) with substitution (15) becomes

$$
\boldsymbol{R}_{\boldsymbol{s}}\left(z_{1} \ldots z_{\boldsymbol{s}} ; q\right)=\prod_{j=1}^{s}\left[1-\alpha_{1}(q) \cdot\left(\varphi_{1}\left(z_{j} ; j\right)-1\right)\right] \text {. }
$$

For a general stationary arrival process, as described in paragraph (2.1.a), we can substitute

$$
\alpha_{s-\lambda}(q \ldots q) \text { for }\left[\alpha_{1}(q)\right]^{s-\lambda}
$$

after having expanded expression (25). Finally, with the new expressions for $R_{s}$ and $N_{s}$, expressions (17) and (18) are still valid if we apply substitution (15). However, it may be very useful to note that the result is not changed if we replace

$$
\alpha_{s-\lambda}\left(-\sum_{\nu=1}^{\lambda+1} z_{\nu}, \ldots,-\sum_{\nu=1}^{s} z_{\nu}\right)
$$

in expression (18) by

$$
\alpha_{s-\lambda}\left(-\sum_{\nu=1}^{k_{\lambda+1}} z_{\nu}, \ldots, \sum_{\nu=1}^{k_{s}} z_{\nu}\right)
$$

To check this equivalence, we come back to the preceding expressions, related to (17) and (18) and leading to relations (30) through (33) in Le Gall [3], to be applied in the above expression (16) in order to satisfy the factorization method. It follows that the successive residues for $\zeta_{i}=z_{i}(i=s, s-1, \ldots, 1)$ lead to the application of $\alpha_{s-\lambda}$ to expression (16). The successive residues at the respective poles $z_{i}=-q-\sum_{\nu=1}^{i}$, for $i=s, s-1, \ldots, 1$, lead to the expression $\alpha_{s-\lambda}(q \ldots q)$, as with the above expression for $\alpha_{s-\lambda}$. We find again expression (25) or a more general expres- 
sion corresponding to expression (11). In other words, the singular points are not changed, and the factorization method is still applicable. The new expression for the functional $\mathrm{E} e^{-q \tau}$ does not change its value, since we know that the solution is unique to determine the $\mathrm{G} / \mathrm{G} / \mathrm{s}$ queue.

Now, we shall use the symmetry in expression (17), which leads to the following substitutions:

$$
\begin{aligned}
& {\left[\varphi_{1}\left(z_{j} ; j\right)-1\right] \cdot \alpha_{s-\lambda}\left(-\sum_{\nu=1}^{k_{\lambda+1}+1} z_{\nu}, \ldots,-\sum_{\nu=1}^{k_{j}} z_{\nu}, \ldots,-\sum_{\nu=1}^{k_{s}} z_{\nu}\right)} \\
& =\frac{\varphi_{1}\left(z_{j} ; j\right)-1}{z_{j}} \cdot\left(z_{j}\right) \cdot \alpha_{s-\lambda}\left(-\sum_{\nu=1}^{k_{\lambda+1}} z_{\nu}, \ldots,-\sum_{\nu=1}^{k_{j}} z_{\nu}, \ldots,-\sum_{\nu=1}^{k_{s}} z_{\nu}\right)
\end{aligned}
$$

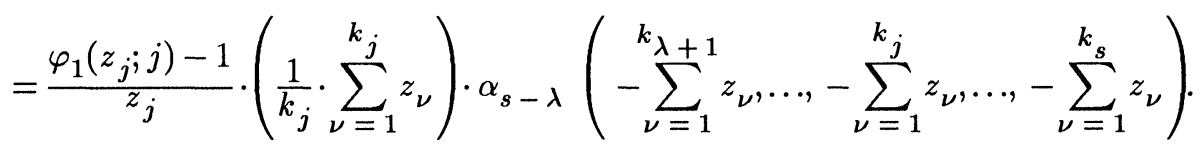

Expression (16) leads to the expression:

$$
\frac{\varphi_{1}\left(z_{j} ; j\right)-1}{z_{j}} \cdot\left(\frac{q}{k_{j}}\right) \cdot \alpha_{s-\lambda}(q \ldots q) .
$$

Finally, considering every variable $z_{j}(j>\lambda)$, we find that (16) reduces to the expression:

$$
\left[\prod_{j=\lambda+1}^{s}\left(\frac{q}{k_{j}}\right)\right] \cdot \alpha_{s-\lambda}(q \ldots q)
$$

instead of $\alpha_{s-\lambda}(q \ldots q)$. Property 1 is satisfied and, finally, we may use expression (9).

Property 2 (The distribution of $\tau$, the queueing delay of the non-symmetrical $\mathrm{G} / \mathrm{G} / \mathrm{s}$ queue): In a stationary regime, if $\boldsymbol{w}(j)$ is the queueing delay of an arbitrary delayed customer served by server $\boldsymbol{j}$ behaving under Property 1 , the queueing delay of an arbitrary delayed customer served by a non-symmetrical (or symmetrical) multiserver is

$$
\tau=\operatorname{Min}[w(1), \ldots, w(s)]
$$

and the expression for the functional $\mathrm{E}\left[e^{-q \tau}\right]$ may be deduced from expression (9).

From the notation in formula (11) in [3], using Pollaczek's formula in [5], for $\operatorname{Re}(q) \geq 0$, we may write

$$
\left\{\begin{array}{c}
\mathrm{E}\left[e^{-q w(j)}\right]=\operatorname{Exp}\left\{\frac{-1}{2 \pi i} \cdot \int_{+0}\left[\frac{1}{q+\zeta}-\frac{1}{\zeta}\right] \cdot \log K(\zeta) \cdot d \zeta\right\} \\
\text { with } K(\zeta)=1-\frac{\alpha_{1}(-\zeta)}{k_{j}} \cdot\left[\varphi_{1}(\zeta ; j)-1\right]
\end{array}\right.
$$

where $\boldsymbol{k}_{\boldsymbol{j}}$ is defined by expression (21). In particular, when $|\boldsymbol{q}|$ increases indefinitely, we obtain, for the probability of no delay of this server equivalent to server $j$ the 
expression

$$
\boldsymbol{Q}_{j}=\operatorname{Exp}\left\{\frac{1}{2 \pi i} \int_{+0} \log \boldsymbol{K}(\zeta) \cdot \frac{d \zeta}{\zeta}\right\}
$$

and for the mean busy period size (= mean number of customers served) we deduce the expression

$$
\boldsymbol{n}_{j}=\frac{1}{Q_{j}}
$$

due to some classical relations. In fact, $\boldsymbol{Q}_{\boldsymbol{j}}$ is the probability of initiating a busy period.

a) Case of the Non-Symmetrical GI/G/s Queue: Taking expressions (9), (28) and (29) into account, we have that the queueing delay $\tau$, of the non-symmetrical GI/G/s queue for an arbitrary delayed customer is given [for $\operatorname{Re}(q)>0$ ] by

$$
\mathbf{E}\left[\boldsymbol{e}^{-\boldsymbol{q} \tau}\right]=1-\frac{1}{(2 \pi i)^{s}} \cdot \int_{+0} \frac{d z_{1}}{z_{1}} \cdots \int_{+0} \frac{d z_{s}}{z_{s}} \cdot\left(\prod_{j=1}^{s} \mathrm{E} e^{-q w(j)}\right) \cdot \frac{q}{q+\sum_{\nu=1}^{s} z_{\nu}}
$$

Expression (35) below will give a simpler expression for the complementary distribution function of the queueing delay, which will be very convenient for numerical calculations.

b) Case of the Non-Symmetrical G/G/s Queue: For the non-symmetrical G/G/s queue, it is much more difficult to expand the terms between brackets in expression (32), since servers are mutually dependent through the arrival process. Finally, it is simpler to proceed in expressions (17) and (18) with the substitutions (15). But the expression in the distribution function of the queueing delay is much more intricate than that of the $\mathrm{GI} / \mathrm{G} / \mathrm{s}$ queue.

\subsection{The Busy Period}

The busy period corresponds to the $s$ servers being busy simultaneously. To evaluate the probability $\boldsymbol{Q}_{\mathbf{0}}$ that the multiserver initiates a busy period, we may note that, among a great number $N$ of successive arrivals, the mean number $N \cdot Q_{0}$ initiates a busy period of the multiserver and the mean number $N \cdot\left[Q_{j} / k_{j}\right]$ corresponds to server $j$ due to Property 1 . We deduce the relations:

$$
\boldsymbol{Q}_{\mathbf{0}}=\sum_{j=1}^{s} \frac{Q_{j}}{k_{j}} \text { and } \frac{1}{\boldsymbol{n}}=\sum_{j=1}^{s} \frac{1}{\boldsymbol{n}_{\boldsymbol{j}} \cdot k_{j}}
$$

due to expression (31), with $\boldsymbol{n}$ being the mean busy period size of the multiserver, and $\boldsymbol{k}_{\boldsymbol{j}}$ being defined by expression (21). In the case of a symmetrical $G / G / \boldsymbol{s}$ queue, where $k_{j}=s$ (see expressions (24)), we have

$$
n_{j}=n, j=1, \ldots, s
$$

\subsection{The Distribution Function}

Let $\boldsymbol{W}$ the queueing delay of an arbitrary (delayed or not delayed) customer and let $\boldsymbol{F}(\boldsymbol{t})$ denote the queueing delay distribution function of a delayed customer. Introduce the complementary function $G(t)=[1-F(t)]$ for the multiserver and introduce 
$G_{j}(t)$ for server $j$.

\subsubsection{Case of the non-symmetrical G/G/s queue}

Let $\mathscr{F}(t)$ denote the queueing delay distribution function of an arbitrary (delayed or not delayed) customer of the multiserver. We have

$$
\mathscr{F}(t)=1-P \cdot G(t)
$$

where $\boldsymbol{P}$ is the probability of delay.

\subsubsection{Case of the non-symmetrical GI/G/s queue}

As we consider the non-symmetrical GI/G/s queue, expression (28) makes say that

$$
G(t)=\prod_{j=1}^{s} G_{j}(t)
$$

since the relation $\tau>t$ needs simultaneously to have $w(j)>t$ for $j=1, \ldots, s$. Expression (29) allows us to evaluate $G_{j}(t)$. Expressions (35) and (36) give for the moments of $W$ :

$$
\mathrm{E}\left(W^{\alpha}\right)=\alpha P \cdot \int_{0}^{\infty} t^{\alpha-1} \cdot G(t) \cdot d t, \quad \alpha=1,2, \ldots
$$

$G_{j}(t)$ and this expression (37) can be easily calculated on a computer.

\subsubsection{The non-symmetrical GI/M/s queue}

From Pollaczek [5] we deduce from expression (29) that

$$
G_{j}(t)=\operatorname{Exp}\left\{-\frac{y_{0}(j)}{T(j)} \cdot t\right\}
$$

where $y_{0}(j)$ is the unique root, for $R(\zeta)>0$, of

$$
K(\zeta)=1-\frac{\alpha_{1}(-\zeta)}{k_{j}} \cdot\left[\varphi_{1}(\zeta ; j)-1\right]=0, \quad \text { with } \varphi_{1}(\zeta ; j)=\frac{1}{1-\frac{\zeta}{T(j)}}
$$

or

$$
\frac{y_{0}(j)}{T(j)}=\frac{1}{1+\frac{1}{k_{j}} \cdot \alpha\left[-y_{0}(j)\right]}
$$

Expressions (30) and (31) become

$$
Q_{j}=y_{0}(j), \text { and } n_{j}=\frac{1}{y_{0}(j)} .
$$

We deduce from expression (21) that

$$
\frac{y_{0}(j)}{T(j)}=\frac{1}{n_{j} \cdot k_{j}} \cdot \frac{s}{\bar{T}}, \quad \text { with } \frac{s}{\bar{T}}=\sum_{j=1}^{s} \frac{1}{T(j)} .
$$

Finally, expression (33) gives us that 


$$
\sum_{j=1}^{s} \frac{y_{0}(j)}{T(j)}=\frac{s}{n \cdot \bar{T}}
$$

where $\boldsymbol{n}$ is the mean busy period size of the multiserver ( = mean number of customers served during a busy period). Consequently, we may write the following simple expression from expressions (36) and (38) for the complementary distribution function of the delayed customer:

$$
G(t)=\operatorname{Exp}\left\{-\frac{s}{n \cdot \bar{T}} \cdot t\right\}
$$

\subsubsection{The non-symmetrical $M / G / s$ queue}

Due to expression (23), we may write

$$
\boldsymbol{Q}_{\boldsymbol{j}}=1-\eta \text { and } n=n_{j}=\frac{1}{1-\eta}
$$

for the mean busy period size $\boldsymbol{n}$ of the multiserver. From Pollaczek [5], we may write

$$
G_{j}(t)=\frac{1-\eta}{\eta} \cdot \sum_{\nu=1}^{\infty} \eta^{\nu} \cdot\left\{1-\left[\int_{0}^{t} \frac{1-F_{1}(u ; j)}{T(j)} \cdot d u\right]^{(\nu)}\right\}
$$

where $[\cdot]^{(\nu)}$ denotes the $k$-fold convolution of the function $[\cdot]$. Finally, $(36)$ gives an intricate expression for the complementary distribution function $G(t)$ of the delayed customer:

$$
G(t)=\prod_{j=1}^{s}\left\{\frac{1-\eta}{\eta} \cdot \sum_{\nu=1}^{\infty} \eta^{\nu} \cdot\left(1-\left[\int_{0}^{t} \frac{1-F_{1}(u ; j)}{\overline{T(j)}} \cdot d u\right]^{(\nu)}\right)\right\}
$$

which can be evaluated numerically on a computer. To use expression (35) we, now, need to define the probability of delay $\boldsymbol{P}$.

\section{The Probability of Delay}

During the busy period ( = the congestion state), server's behavior has been defined in a way quite independent of partial occupancy states. For these states, it follows that a busy period appears exactly as a unique congestion state in the lost call model, with $\boldsymbol{n}$ successive service times handled as if there were a unique arrival, with $\boldsymbol{n}$ being the mean value of the busy period size, i.e., of the number of customers served during this busy period. This fact could not be observed with the classical Markovian methods, and it has not been noted in Pollaczek's equation of [6].

With the lost call model in a stationary mode, let $\boldsymbol{P}_{\boldsymbol{i}}=P_{0} \cdot h(i)$ denote the probability that $i$ servers are busy upon the arrival of an arbitrary customer. The probability of loss is $\boldsymbol{P}_{\boldsymbol{a}}=P_{s}$ with

$$
\frac{1}{\boldsymbol{P}_{\boldsymbol{a}}}=\frac{1}{P_{s}}=1+\frac{1+\ldots+h(s-1)}{h(s)}
$$


due to the normalizing condition $\sum_{i=0}^{s} P_{i}=1$.

With our preceding remark to evaluate the probability of delay $\boldsymbol{P}$, we have to substitute

$$
\boldsymbol{n} \cdot h(s) \text { for } h(s)
$$

We may now conclude.

Property 3 (The probability of delay): For a non-symmetrical G/G/s queue in a stationary regime, the probability of delay is

$$
\boldsymbol{P}=\frac{n \cdot P_{a}}{1+(n-1) \cdot P_{a}},
$$

where $\boldsymbol{P}_{\boldsymbol{a}}$ is the probability of loss in the lost call model and $\boldsymbol{n}$ is the mean value of the busy period size as defined by expression (33).

As already seen in [1] and [2], we know that the evaluation of $\boldsymbol{P}_{\boldsymbol{a}}$ is extremely difficult except in two symmetrical cases: The GI/M/s and $M / G / s$ queues. In particular, for the M/G/s queue we conclude that the delay Erlang formula may be extended for a general service time distribution. In that case, expression (44) gives $\boldsymbol{n}=$ $[1 /(1-\eta)]$. From fact 1$)$ after Property 1 , we know that the $\mathbf{M} / \mathbf{D} / \mathbf{s}$ queue has to be excluded; however, it has already been noted by C. Palm that the delay Erlang formula gives still an excellent approximation.

\section{Numerical Comparisons for Some M/G/s Queues}

Now, we present some numerical comparisons with the results obtained by applying classical Markovian methods to the symmetrical $M / E_{2} / s$ and $M / H_{2} / s$ queues.

\subsection{The Symmetrical $M / E_{2} / s$ Queue}

The service time distribution of any server $j$ is

$$
\left\{\begin{array}{lc}
F_{1}(t)=1-e^{-2 t} \cdot(1+2 t) ; & \varphi_{1}(z)=\frac{1}{\left(1-\frac{z}{2}\right)^{2}} \\
\text { corresponding to } \mathbf{E}(T)=1 ; & C_{s}^{2}=\frac{\operatorname{Var} T}{[\mathrm{E}(T)]^{2}}=\mathbf{0 . 5}
\end{array}\right.
$$

We deduce, for the queueing delay $w(j)$ of any server $j$ when $\operatorname{Re}(z)<0$ :

$$
\left\{\begin{array}{c}
\mathrm{E}\left[e^{z w(j)}\right]-\frac{1-\eta}{1-\eta \cdot \frac{\varphi_{1}(\xi)-1}{\xi}}=\frac{(1-\eta)\left(1-\frac{z}{2}\right)^{2}}{\left(1-\frac{z}{2}\right)^{2}-\eta \cdot\left(1-\frac{z}{4}\right)}=\frac{4(1-\eta)\left(1-\frac{z}{2}\right)^{2}}{\left(z-\beta_{1}\right)\left(z-\beta_{2}\right)} \\
\quad \text { with } \beta_{1}=2\left(1-\frac{\eta}{4}\right)-2 \sqrt{\frac{\eta}{2} \cdot\left(\frac{\eta}{8}+1\right)} \\
\text { and } \beta_{2}=2\left(1-\frac{\eta}{4}\right)+2 \sqrt{\frac{\eta}{2} \cdot\left(\frac{\eta}{8}+1\right)} .
\end{array}\right.
$$

The queueing delay distribution function of this server $j$ is 


$$
\left\{\begin{array}{c}
F_{j}(t)=F_{1}(t)=1-\eta \cdot G_{1}(t) \\
\text { with } G_{1}(t)=(1-\eta) \cdot\left\{\frac{1}{\left(\beta_{2}-\beta_{1}\right)} \cdot \frac{4-\beta_{1}}{\beta_{1}} \cdot e^{-\beta_{1} t}+\frac{1}{\left(\beta_{1}-\beta_{2}\right)} \cdot \frac{4-\beta_{2}}{\beta_{2}} \cdot e^{-\beta_{2} t}\right\} .
\end{array}\right.
$$

From expression (36), for the multiserver, the queueing delay distribution function of the delayed customer is

$$
p=F(t)=1-\left[G_{1}(t)\right]^{s} .
$$

where $\boldsymbol{t}$ is the conditional queueing delay percentile $\boldsymbol{t}(\boldsymbol{p})$.

In Table 1 , we give (for $\eta=0.8$ ) our results concerning $t(p)$ and the probability of delay $\boldsymbol{P}$ from expression (49), for $s=2,5,10,25,50$ and $p=0.5,0.9,0.95,0.99$. For the results given by the Markovian methods (= phase methods), we refer to Table 1 (first part) in Seelen and Tijms [7]. These results appeared as approximated (Markovian) results in our Table 1. The deviation is not significant.

\begin{tabular}{|l|l|l|l|l|l|c|}
\hline & $p$ & 0.5 & 0.9 & 0.95 & 0.99 & $P$ \\
\hline \multirow{3}{*}{$s=2$} & exact & 1.36 & 4.31 & 5.58 & 8.52 & 0.711 \\
\cline { 2 - 7 }$s=5$ & Markov & 1.34 & 4.29 & 5.55 & 8.50 & 0.709 \\
\hline \multirow{3}{*}{$s=10$} & exact & 0.58 & 1.78 & 2.29 & 3.47 & 0.554 \\
\cline { 2 - 7 } & Markov & 0.55 & 1.73 & 2.24 & 3.42 & 0.548 \\
\hline \multirow{3}{*}{$s=25$} & exact & 0.31 & 0.93 & 1.19 & 1.78 & 0.409 \\
\cline { 2 - 7 } & Markov & 0.29 & 0.88 & 1.13 & 1.72 & 0.402 \\
\hline \multirow{3}{*}{$s=50$} & exact & 0.13 & 0.40 & 0.51 & 0.75 & 0.209 \\
\cline { 2 - 7 } & Markov & 0.12 & 0.36 & 0.47 & 0.70 & 0.203 \\
\cline { 2 - 7 } & exact & 0.07 & 0.21 & 0.27 & 0.40 & 0.087 \\
\hline \multirow{2}{*}{$s a r k o v$} & 0.06 & 0.19 & 0.24 & 0.36 & 0.084 \\
\hline
\end{tabular}

Table 1: The symmetrical $\mathrm{M} / \mathrm{E}_{2} / \mathrm{s}$ queue

1) Results a) the conditional queueing delay percentile $t(p)$ for the delayed customer

b) probability of delay: $\boldsymbol{P}$

2) Parameters a) traffic intensity per server: $\boldsymbol{\eta}=\mathbf{0 . 8}$

b) service time distribution from expression (50): $\mathbf{E}(\boldsymbol{T})=1$, $C_{s}^{2}=0.5$

3) Calculations a) "exact": Section 5.1

b) "Markov": Phase method

\subsection{The Symmetrical $\mathrm{M} / \mathrm{H}_{2} / \mathrm{s}$ Queue}

The service time distribution of any server $j$ is:

$$
\left\{\begin{array}{c}
F_{1}(t)=0.5 \cdot\left[1-e^{-b_{1} t}\right]+0.5 \cdot\left[1-e^{-b_{2} t}\right], b_{1}=\frac{2}{2-\sqrt{2}} ; b_{2}=\frac{2}{2+\sqrt{2}} \\
\text { corresponding to } \mathbf{E}(T)=1, \quad C_{s}^{2}=\frac{\operatorname{Var} T}{[\mathrm{E}(T)]^{2}}=2 .
\end{array}\right.
$$


We let

$$
\left\{\begin{array}{l}
\beta_{1}=\frac{b_{1}+b_{2}-\eta}{2}+\sqrt{\frac{\left(b_{1}+b_{2}-\eta\right)^{2}}{4}-b_{1} \cdot b_{2} \cdot(1-\eta)} \\
\beta_{2}=\frac{b_{1}+b_{2}-\eta}{2}-\sqrt{\frac{\left(b_{1}+b_{2}-\eta\right)^{2}}{4}-b_{1} \cdot b_{2} \cdot(1-\eta)}
\end{array}\right.
$$

As for expression (52), the complementary queueing delay distribution function of any server $\boldsymbol{j}$ is

$$
G_{1}(t)=\frac{\beta_{2}-1+\eta}{\beta_{2}-\beta_{1}} \cdot e^{-\beta_{1} t}+\frac{\beta_{1}-1+\eta}{\beta_{1}-\beta_{2}} \cdot e^{-\beta_{2} t}
$$

Now, table 2 uses expressions (53) and (56) to give the new values of $\boldsymbol{t}(\boldsymbol{p})$ and $\boldsymbol{P}$ corresponding to the same values of parameters $\boldsymbol{\eta}, \boldsymbol{s}$ and $\boldsymbol{p}$ as in Table 1 . For the approximated (Markovian) results we refer to Table 1 (second part) in Seelen and Tijms [7]. The deviation is not significant either.

\begin{tabular}{|l|l|l|l|l|l|c|}
\hline & $p$ & 0.5 & 0.9 & 0.95 & 0.99 & $\boldsymbol{P}$ \\
\hline \multirow{3}{*}{$s=2$} & exact & 2.47 & 8.65 & 11.30 & 17.43 & 0.711 \\
\cline { 2 - 7 }$s=5$ & Markov & 2.36 & 8.82 & 11.62 & 18.11 & 0.715 \\
\hline \multirow{3}{*}{$s=10$} & exact & 0.89 & 3.35 & 4.41 & 6.86 & 0.554 \\
\cline { 2 - 7 } & Markov & 0.89 & 3.41 & 4.53 & 7.12 & 0.562 \\
\hline \multirow{3}{*}{$s=25$} & exact & 0.40 & 1.58 & 2.11 & 3.34 & 0.409 \\
\cline { 2 - 7 } & Markov & 0.43 & 1.63 & 2.19 & 3.48 & 0.418 \\
\hline \multirow{3}{*}{$s=50$} & exact & 0.15 & 0.56 & 0.76 & 1.22 & 0.209 \\
\cline { 2 - 7 } & Markov & 0.16 & 0.60 & 0.81 & 1.31 & 0.216 \\
\cline { 2 - 7 } & exact & 0.07 & 0.26 & 0.34 & 0.55 & 0.087 \\
\cline { 2 - 7 } & Markov & 0.08 & 0.28 & 0.38 & 0.61 & 0.090 \\
\hline
\end{tabular}

Table 2: The symmetrical $\mathrm{M} / \mathrm{H}_{2} / \mathrm{s}$ queue

1) Results a) the conditional queueing delay percentile $t(p)$ for a delayed customer

b) probability of delay: $\boldsymbol{P}$

2) Parameters a) traffic intensity per server: $\boldsymbol{\eta}=\mathbf{0 . 8}$

b) service time distribution from expression (54): $\mathbf{E}(T)=1$, $C_{s}^{2}=2$

3) Calculations

a) "exact": Section 5.2

b) "Markov": Phase method

Tables 3 (for $\eta=0.5$ ) and 4 (for $\eta=0.9$ ) give some comparisons between values of the mean queueing delay $\bar{W}$ of an arbitrary (delayed or not delayed) customer, given by calculation and by traffic simulation. Now, the service time distribution of any server $j$ is 


$$
\left\{\begin{array}{c}
F_{1}(t)=a_{1} \cdot\left[1-e^{-b_{1} t}\right]+\left(1-a_{1}\right) \cdot\left[1-e^{-b_{2} t}\right] \\
\text { with } a_{1}=\frac{50 \cdot(n-1)}{50 \cdot n+31}, \quad b_{1}=10, \quad b_{2}=\frac{10 \cdot\left(1-a_{1}\right)}{10-a_{1}} \\
\text { corresponding to } \mathbf{E}(T)=1, C_{s}^{2}=\frac{\operatorname{Var} T}{[\mathrm{E}(T)]^{2}}=n
\end{array}\right.
$$

\begin{tabular}{|l|l|l|l|}
\cline { 2 - 4 } \multicolumn{1}{c|}{} & $n$ & 5 & 10 \\
\hline \multirow{3}{*}{$s=2$} & $C$ & 0.98 & 1.76 \\
\cline { 2 - 4 } & $S$ & 1.0 & 1.77 \\
\hline \multirow{3}{*}{$s=5$} & $C$ & 0.14 & 0.24 \\
\cline { 2 - 4 } & $S$ & 0.15 & 0.25 \\
\hline
\end{tabular}

Table 3: The symmetrical $\mathrm{M} / \mathrm{H}_{2} / \mathrm{s}$ queue

The mean queueing delay $\bar{W}$ of an arbitrary customer

1) Comparisons between calculations (expression (58)), line $C$ and simulations, line $\mathbf{S}$.

2) Parameters a) traffic intensity per server: $\boldsymbol{\eta}=\mathbf{0 . 5}$

b) service time distribution from expression (57): $\mathbf{E}(T)=1, C_{s}^{2}=n$

\begin{tabular}{|l|l|l|l|}
\cline { 2 - 4 } \multicolumn{1}{c|}{} & $n$ & 5 & 10 \\
\hline \multirow{3}{*}{$s=5$} & $C$ & 4.5 & 8.3 \\
\cline { 2 - 4 } & $S$ & 4.6 & 8.7 \\
\hline \multirow{3}{*}{$s=10$} & $C$ & 1.9 & 3.3 \\
\cline { 2 - 4 } & $S$ & 2.0 & 3.2 \\
\hline
\end{tabular}

Table 4: The symmetrical $\mathrm{M} / \mathrm{H}_{2} / \mathrm{s}$ queue

The mean queueing delay $\bar{W}$ of an arbitrary customer

1) Comparisons between calculations (expression (58)), line $C$ and simulations, line $\mathbf{S}$.

2) Parameters a) traffic intensity per server: $\boldsymbol{\eta}=\mathbf{0 . 9}$

b) service time distribution from expression (57): $\mathbf{E}(T)=1, C_{s}^{2}=n$

On applying expressions (55) and (56), we deduce from (37):

$$
\overline{\boldsymbol{W}}=\boldsymbol{P} \cdot \int_{0}^{\infty}\left[G_{1}(t)\right]^{s} \cdot d t
$$

where $\boldsymbol{P}$ is given by expression (49). Tables 3 and 4 consider the cases $n=5$ and 10 . Taking the accuracy of simulations and of calculations of (58) into account, the results given by traffic simulations and by calculations are in good agreement. 


\section{Conclusion}

We characterized non-symmetrical (and symmetrical) G/G/s queues by three simple properties which lead to very simple numerical calculations, at least for the GI/G/s queues. The deviation of the numerical results with those of classical, approximated, Markovian methods is not significant, and our numerical results are in good agreement with those given by traffic simulations.

\section{References}

[1] Le Gall, P., General telecommunications traffic without delay, Proc. International Teletraffic Congress, ITC 8 (Melbourne, Australia, Nov. 1976) 125.

[2] Le Gall, P., Sur le problème du trafic téléphonique général, direct et sans attente, Annales Telec., Paris (Sept.-Oct.) 34:9-10 (1979), 459-468.

[3] Le Gall, P., The stationary G/G/s queue, JAMSA 11:1 (1998), 59-71.

[4] Loynes, R.M., The stability of a queue with nonindependent interarrival and service times, Proc. Cambridge Philos. Soc. 58 (1962), 494-520.

[5] Pollaczek, F., Problèmes stochastiques posés par le phénomène de formation d'une queue d'attente à un guichet et par des phénomènes apparentés, Mémorial des Sciences Mathématiques, Gauthier-Villars, Paris CXXXVI (1957). ( = GI/G/1 queue; in French).

[6] Pollaczek, F., Théorie analytique des problèmes stochastiques relatifs à un groupe de lignes téléphoniques avec dispositif d'attente, Mémorial des Sciences Mathématiques, Gauthier-Villars, Paris CL (1961). (=GI/G/s queue; in French).

[7] Seelen, L.P. and Tijms, H.c., Approximations to the waiting time percentiles in the M/G/c queue, Proc. Intern. Teletraffic Cong. ITC-11 (Kyoto), Sept. 1985, 1.4-4. 


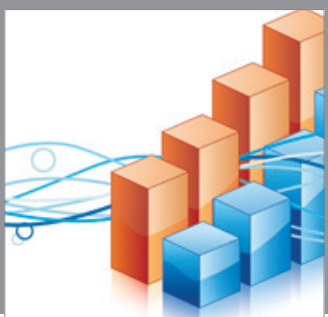

Advances in

Operations Research

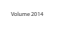

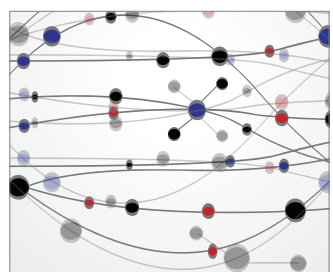

\section{The Scientific} World Journal
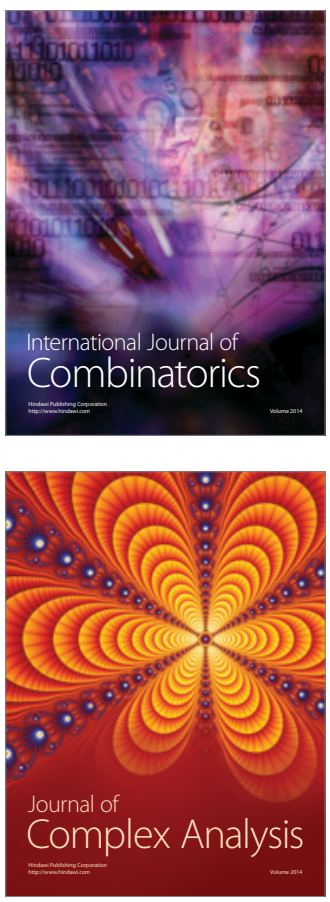

International Journal of

Mathematics and

Mathematical

Sciences
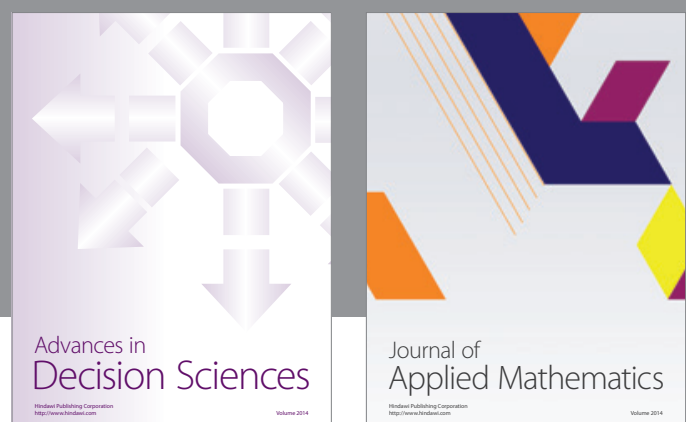

Journal of

Applied Mathematics
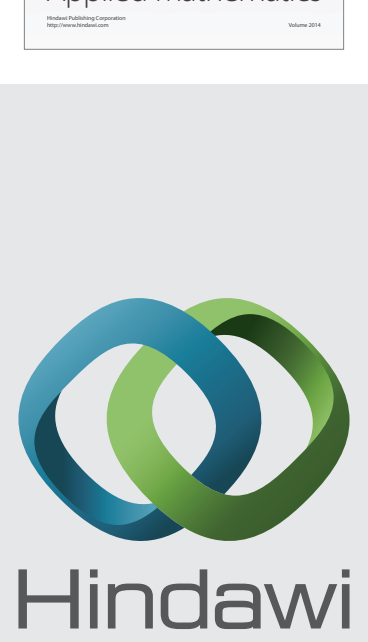

Submit your manuscripts at http://www.hindawi.com
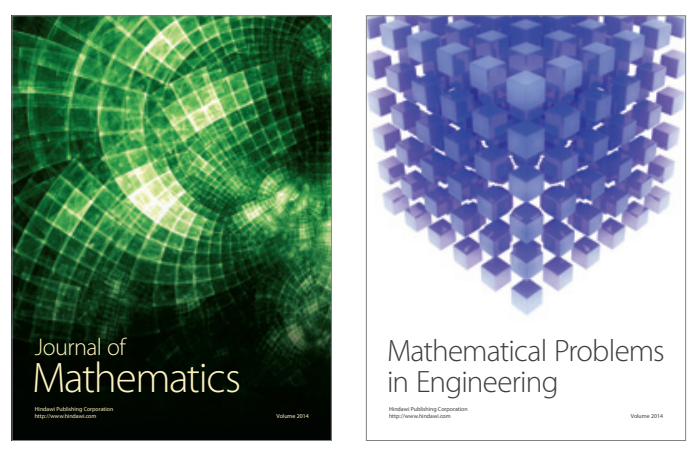

Mathematical Problems in Engineering
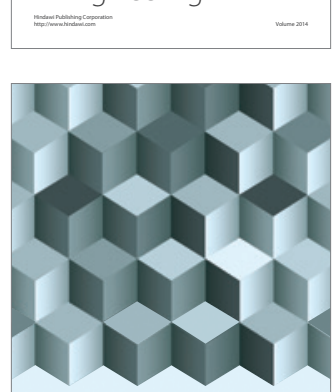

Journal of

Function Spaces
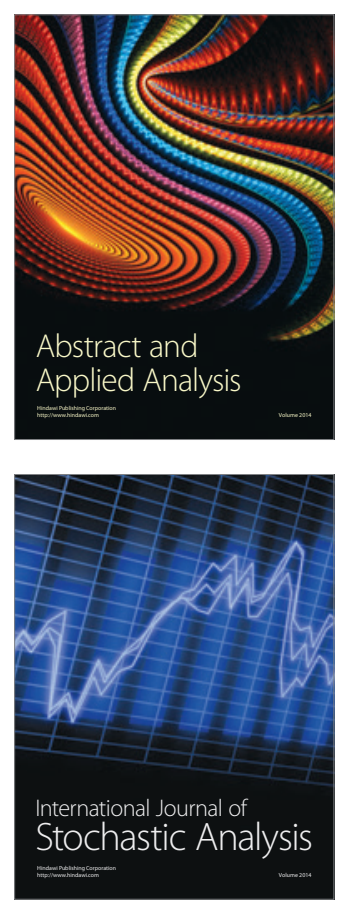

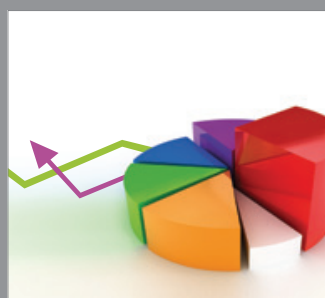

ournal of

Probability and Statistics

Promensencen
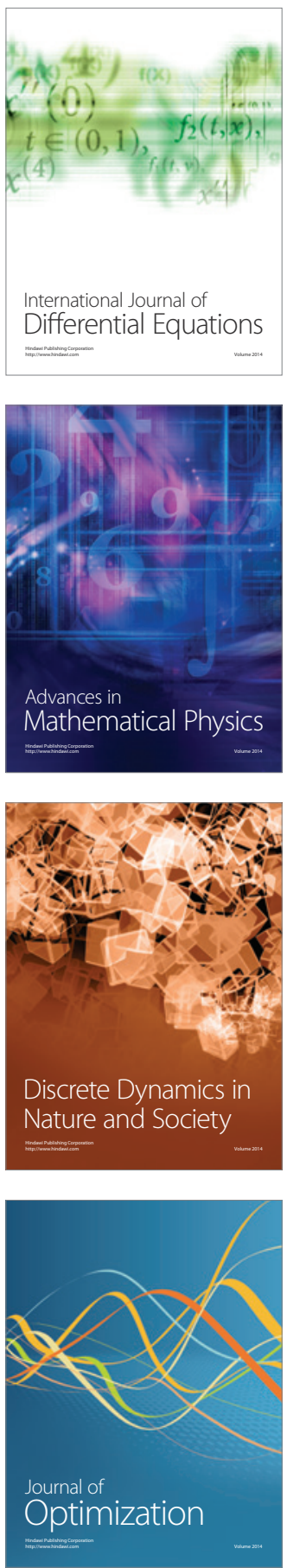